\title{
The role of fouling in optimizing direct-flow filtration module design
}

\author{
Mylène Wang ${ }^{\mathrm{a}}$, Sourav Mondal ${ }^{\mathrm{b}}$, Ian M. Griffiths ${ }^{\mathrm{b}, *}$ \\ ${ }^{a}$ ENSTA ParisTech, 828, Boulevard des Maréchaux 91120 Palaiseau, France \\ ${ }^{b}$ Mathematical Institute, Andrew Wiles Building, University of Oxford, OX2 6GG, UK
}

\begin{abstract}
The local transmembrane pressure (TMP) distribution plays a crucial role in the performance of hollow-fibre microfiltration and ultrafiltration membrane devices. A direct-flow filter comprises an array of hollow fibres encased within a single module. The TMP in each hollow fibre is dependent on the spacing between neighbouring fibres, and evolves with time due to pore clogging by contaminants (fouling). We consider an idealized set-up in which the fibres are undeformable and equally spaced within the device, and study the impact of the pore-blocking phenomena on the TMP during the filtration process. The model is used to evaluate the optimum inter-fibre spacing that maximizes the fluid processed either after a prescribed time or before the filter blocks and its dependence on the membrane permeability and the fouling rate. We show that significant improvements can be made on the operating efficiency of a direct-flow device through careful choice in the fibre spacing during fabrication.
\end{abstract}

Keywords: Membrane, filtration, fouling, hollow fibre, module design.

\section{Introduction}

The design of filtration modules is key to membrane technology: the performance and economics of a membrane process is dependent on the art and sophistication of the device manufacture [1]. As a result, exploring the scope of module design optimization for commercial exploitation of membrane systems has become increasingly important $[2,3]$. The filtration technique may be classified by a series of methods. In classical dead-end filtration, the flow and filtration direction both occur normal to the membrane surface. However, in cross-flow filtration the feed flows parallel to the surface of the membrane with fluid filtration occurring normal to the flow [4]. Each filtration technique has its merits and downfalls. Crossflow filtration is the de facto method when processing high volumes of fluid. However, it is complex in operation and capital intensive. On the other hand, deadend filtration is straightforward to implement but cannot compete when it comes to processing high volumes of fluid or high contaminant concentrations. Direct-flow filtration offers an efficient combination of the benefits of both dead-end and cross-flow filtration. This is achieved by capping the end of a crossflow device so that all the fluid is forced to pass through the walls. Direct-flow filtration offers superior performance in situations where low contaminant concentrations as well as moderate fluid volumes are processed and the product quality as well as sim-

\footnotetext{
*Corresponding author

Email address: ian.griffiths@maths.ox.ac.uk (Ian M. Griffiths)
}

plicity is preferred, such as in applications related to biopharmaceuticals, virus separation and sterile filtration.

The main quantity of interest in any filtration scenario is the rate at which fluid is processed, or the throughput. This increases with transmembrane pressure (TMP) and filtration area. In a typical industrial application, the filtration area in a direct-flow device is increased by bundling together many hollow fibres with porous walls, or lumen, into a single cartridge whose end is blocked, so the inlet is through the hollow-fibre core and the outlet is across the porous wall (figure 1a). However, if the fibres are packed too close the TMP across significant proportions of the fibre walls is reduced. As a result, despite the increased membrane area, continuing to pack in more fibres causes the overall rate of fluid processed by the device to fall $[5,6]$. A key question to ask is thus how many fibres should we pack into a single cartridge to maximize the overall fluid processing rate [7].

A mathematical model of the direct-flow process has been proposed by Hurwitz [8] by considering the fluid transport inside a single porous tube with a capped end. The flow was determined in the asymptotic limits of low permeability and low Reynolds number. The effect of deposition of the particles on the surface in a single hollowfibre dead-end filtration set-up has been examined (see, for example, $[9,10]$ ), while the blocking behaviour in a membrane when channels are packed adjacent to one another has also been investigated [11]. While emphasis has been placed on the spatio-temporal distribution of the local pressure as a function of the length and diameter of the fibres, the effect of the proximity of the neighbouring tubes was not considered. 
Herterich et el. [6] focus specifically on the variations in TMP in a direct-flow device by accounting for neighbouring fibres, and show that a packing fraction that maximizes the processed fluid exists. Here the membrane permeability is assumed to be uniform and unchanged as the fluid is filtered. In practice, however, as time progresses the membrane will become blocked due to the particles that become trapped within. This reduces the wall permeability and thus the ease with which subsequent fluid may be processed. Furthermore, since the flow across the membrane varies with axial position this will lead to spatial inhomogeneities in the wall permeability. When operating at a fixed pressure difference across the device the effect of this membrane blocking, or fouling, presents itself through a continual decline in the rate at which the filtered fluid is generated. Such fouling behaviour poses significant additional complications when addressing the question of how best to arrange the hollow fibres within a direct-flow device.

In this paper we develop a mathematical model to determine the optimum fibre packing criteria taking into account the membrane fouling due to particle clogging with filtration time. We discuss what is meant by efficiency in a direct-flow device and show that this is not necessarily determined by a single measurable outcome. We identify a family of metrics that are able to classify system optimization for a given filtration challenge, and show how the results are affected by the blocking behaviour.

\section{Model development}

\subsection{Set-up}

A direct-flow device is composed of a series of fibres packed within a larger vessel (figure 1a). For simplicity we consider a slice through such a device, analysing an analogous two-dimensional problem. (Such an assumption has been justified in [6].) We consider fluid entering a single $2 \mathrm{D}$ channel, of length $\tilde{L}$ and width $\tilde{H}$, with porous walls of permeability $\tilde{\kappa}$, and a capped end (figure $1 \mathrm{~b}$ ). We use a Cartesian coordinate system $(\tilde{x}, \tilde{y})$ to represent the system, where $\tilde{x}$ denotes the distance along the channel and $\tilde{y}$ the direction perpendicular to the permeable channel walls.

Fluid enters the channel (Region 1) and passes through the membrane side walls into the permeate region (Region 2 ). The flow in Region 2 is influenced by the proximity of the surrounding channels in the direct-flow device. (For a single channel Region 2 would be a quiescent bath.) Since the end of the channel is blocked, all of the fluid must eventually flow from Region 1, through the permeable walls, into Region 2 . We consider an array of channels with a centre-centre separation $2(\tilde{H}+\tilde{D})$ and so impose a symmetry condition at $\tilde{x}= \pm(\tilde{H}+\tilde{D})$. This symmetry means that we need only solve the system for $(\tilde{x}, \tilde{y})$ $\in([0, \tilde{L}],[0, \tilde{H}+\tilde{D}])$. We denote the membrane thickness be $\tilde{h}$ so that Region 1 occupies $(\tilde{x}, \tilde{y}) \in([0, \tilde{L}],[0, \tilde{H}])$ and Region 2 occupies $(\tilde{x}, \tilde{y}) \in([0, \tilde{L}],[\tilde{H}+\tilde{h}, \tilde{H}+\tilde{D}])$. The fluid

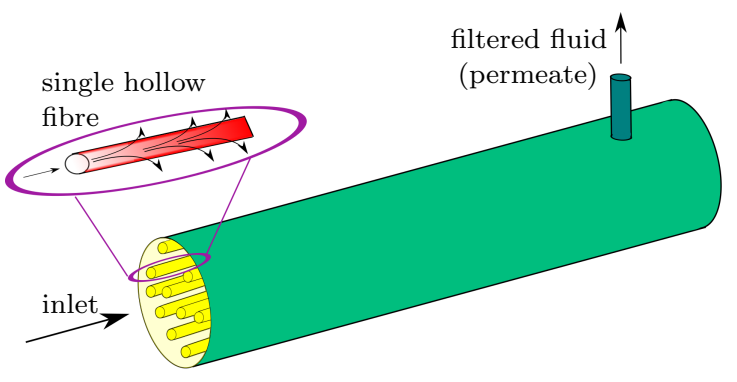

(a)

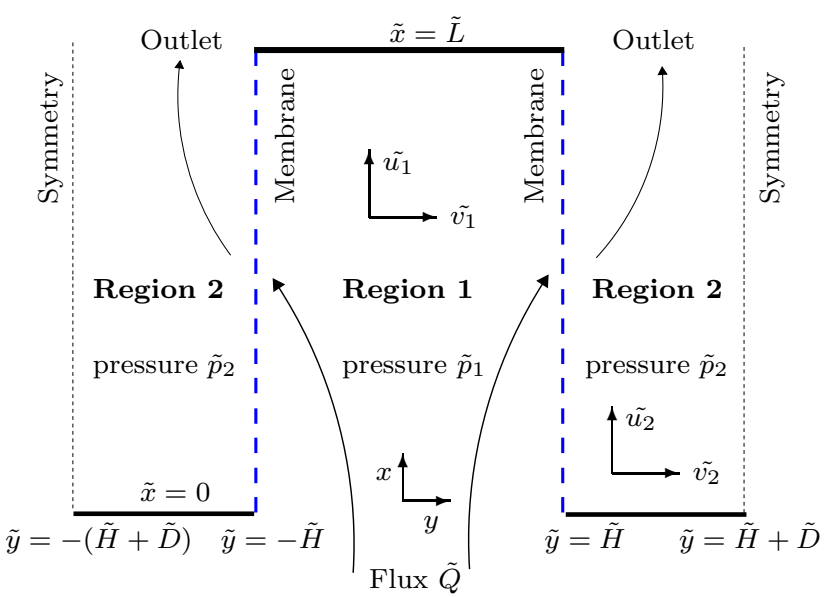

(b)

Figure 1: (a) Schematic of a hollow-fibre direct-flow filtration device. Multiple permeable fibres are encased within a single unit. (b) Schematic of the flow within a single hollow fibre. The solid lines denote impermeable walls, the blue dashed lines are permeable membrane walls, and the dotted line indicates symmetry in the flow. Flow enters from the bottom and passes through the permeable walls. Beyond the capped end the fluid continues to an outlet following the black arrows. The inside of the membranewalled channel is denoted by Region 1 and outside is Region 2 . 
velocity field in the $(\tilde{x}, \tilde{y})$ directions in Region $i(=1,2)$ is $\boldsymbol{u}_{i}=\left(\tilde{u}_{i}(\tilde{x}, \tilde{y}), \tilde{v}_{i}(\tilde{x}, \tilde{y})\right)$, while the pressure is $\tilde{p}_{i}$.

We consider an idealized set-up in which the membrane is composed of uniformly spaced pores of initially constant radius $\tilde{r}_{0}$. In practice it is inevitable that there will be some randomness in the inter-fibre spacing, but we expect that the results of the analysis we present will provide the averaged behaviour over a series of such devices. In addition, the model we present here may be generalized to describe a system that is characterized by a mean interfibre spacing and standard deviation about this mean. We assume that the fluid in Region 1 is uniformly laden with particles of radius $\tilde{a}<\tilde{r}_{0}$. As the fluid passes through the membrane the particles are filtered out by adhering to the pore walls with a finite probability. We assume, for simplicity, that these particles are neutrally buoyant, so that the density of the fluid is unchanged when the particles are removed, and that the viscosity is unaffected by the presence of the particles (though our analysis readily generalizes to account for such variations).

\subsection{Fluid equations}

Typically, for hollow-fibre membrane module configurations, $\tilde{L}=1.5 \mathrm{~m}$ and $2 \tilde{H}=0.7-1 \mathrm{~mm}$, so that the aspect ratio, $\delta=H / L=O\left(10^{-3}\right)$. The membrane is thin (e.g. typical thicknesses $h \sim 10 \mu \mathrm{m}[12]$ and so $\tilde{h} / \tilde{H} \sim 10^{-3}$ ) and we can ignore the behaviour within the porous walls when solving for the flow. The Reynolds number for the channel flow is defined as $\operatorname{Re}=2 \tilde{\rho} \tilde{H} \tilde{U} / \tilde{\mu}$, where $\tilde{U}$ is the typical axial velocity, $\tilde{\mu}$ is the fluid viscosity and $\tilde{\rho}$ is the fluid density. For water, this gives $R e \approx 20$, and a reduced Reynolds number $\delta R e=O\left(10^{-2}\right) \ll 1$ [7]. Since the reduced Reynolds number is small and the channel is thin, the fluid flow obeys the lubrication equations [13].

We non-dimensionalize distances with the channel length and exploit the small aspect ratio $\delta=\tilde{H} / \tilde{L} \ll 1$. We scale the velocities with the typical axial velocity, the pressure assumes the conventional lubrication scaling, and we consider the problem on the advective timescale:

$$
\begin{aligned}
& \tilde{x}=\tilde{L} x, \quad \tilde{y}=\delta \tilde{L} y, \quad \tilde{t}=\frac{\tilde{L}}{\tilde{U}} t, \\
& \tilde{u}_{i}=\tilde{U} u_{i}, \quad \tilde{v}_{i}=\delta \tilde{U} v_{i} \quad \tilde{p}_{i}=\frac{\tilde{\mu} \tilde{U}}{\delta^{2} \tilde{L}} p_{i} .
\end{aligned}
$$

In Region $i(=1,2)(0<x<1)$ the fluid satisfies the lubrication equations

$$
\begin{aligned}
\frac{\partial u_{i}}{\partial x}+\frac{\partial v_{i}}{\partial y} & =0 \\
\frac{\partial p_{i}}{\partial x} & =\frac{\partial^{2} u_{i}}{\partial y^{2}}
\end{aligned}
$$

where $p_{i}=p_{i}(x, t)$ is independent of $y$. Region 1 occupies the dimensionless domain $(x, y) \in([0,1],[0,1])$ and Region 2 occupies the dimensionless domain $(x, y) \in([0,1],[1,1+$ $\ell]$ ), where $\ell=\tilde{D} / \tilde{H}$.
We apply a constant pressure difference across the filter device so that

$$
p_{1}(0, t)=1 \quad p_{2}(1, t)=0 .
$$

We also apply appropriate symmetry conditions

$$
\begin{aligned}
\frac{\partial u_{1}}{\partial y}(x, 0, t) & =0, & & \frac{\partial u_{2}}{\partial y}(x, 1+\ell, t)=0, \\
v_{1}(x, 0, t) & =0, & & v_{2}(x, 1+\ell, t)=0 .
\end{aligned}
$$

The end of Region 1 and the beginning of Region 2 are capped so we have zero perpendicular flow:

$$
u_{1}(1, y, t)=u_{2}(0, y, t)=0 .
$$

In this model, there is a transverse flow, $v_{1}$, at the capped end, $x=1$. In reality, there is a no-slip condition here $\left(v_{1}=0\right)$. As a result of exploiting the slenderness of the system geometry, the order of the system is reduced and we do not need to impose this additional constraint. However, this no-slip condition influences the flow only in a boundary layer of size $\delta$ near the capped end (where we would have to solve the full Stokes equations, as considered in [8]) and so we are able to neglect this effect in our model.

At the membrane we assume a filtration velocity that is proportional to the transmembrane pressure difference and the permeability of the membrane (i.e., Darcy flow) [14]. This gives

$$
v_{1}(x, 1, t)=v_{2}(x, 1, t)=\kappa(x, t)\left(p_{1}(x, t)-p_{2}(x, t)\right),
$$

where

$$
\kappa=\frac{\tilde{\kappa}}{\delta^{2} \tilde{L} \tilde{h}},
$$

is a dimensionless measure of the permeability. Here we shall assume $\kappa=O(1)$ to obtain the richest system behaviour. (The limits $\kappa \ll 1$ and $\kappa \gg 1$ will be subsets of this regime.) In general, at the permeable wall there is a tangential slip velocity, whose magnitude is determined by a Neumann boundary condition such as that given in [15]. However, it has been found that this slip is not significant for a wide range of membranes $[16,17]$ and so here, for simplicity, we shall assume a no-slip boundary condition:

$$
u_{1}(x, 1, t)=u_{2}(x, 1, t)=0
$$

For a given permeability, $\kappa$, the system (2) subject to conditions (3)-(8) determines the velocities $u_{i}, v_{i}$ and pressures $p_{i}, i=1,2$. In the following section we derive an equation for the evolution of the permeability with time due the accretion of particles within the membrane pores, to close the system. 


\subsection{Fouling and solute polarization}

On a microscopic level, we assume that the membrane is composed of an array of circular pores, of radius $r(t)$. We assume that the membrane is undeformed under application of a transmembrane pressure. The dimensional flux through a pore of radius $\tilde{r}$ is given by Poiseuille's law as [18]

$$
\tilde{Q}_{p}=\frac{\pi\left(\tilde{p}_{1}-\tilde{p}_{2}\right) \tilde{r}^{4}}{8 \tilde{\mu} \tilde{h}} .
$$

The net volumetric flux per unit area of membrane is therefore given by

$$
\tilde{Q}=\tilde{N}_{A} \tilde{Q}_{p}
$$

where $\tilde{N}_{A}$ is the number of pores per unit area of membrane. Equation (7) (expressed in dimensional terms) also gives that

$$
\tilde{Q}=\frac{\tilde{\kappa}}{\tilde{\mu} \tilde{h}}\left(\tilde{p}_{1}-\tilde{p}_{2}\right)
$$

and so by equating (10) and (11) we find that the permeability is given by

$$
\tilde{\kappa}=\frac{\pi \tilde{N}_{A} \tilde{r}^{4}}{8}
$$

The radius of the pores will shrink in response to the internal deposition of particles and, on average, for the simplest model for radial contraction, the pore radius following the deposition of $n(t) \geq 1$ spherical particles of radius $\tilde{a}<\tilde{r}$ is

$$
\tilde{r}(\tilde{t})=\sqrt{\tilde{r}(0)^{2}-\frac{4}{3 h} \tilde{a}^{3} n(\tilde{t})} .
$$

This type of blocking law is commonly called standard blocking (see, for example, [19]).

We assume that the concentration of particles within the fluid, $\tilde{c}$, is constant and so the rate at which particles adhere to a pore will be proportional to the flux of fluid through the pore (given by (9)) multiplied by the particle concentration $\tilde{c}$ and the probability that a particle will adhere to the wall, $\lambda$ :

$$
\frac{\mathrm{d} n}{\mathrm{~d} \tilde{t}}=\frac{\pi \lambda\left(\tilde{p}_{1}-\tilde{p}_{2}\right) \tilde{r}^{4} \tilde{c}}{8 \tilde{\mu} \tilde{h}} .
$$

Thus, upon differentiation of (12) and use of (13) and (14) we arrive at the evolution equation for the dimensionless wall permeability,

$$
\frac{\partial \kappa}{\partial t}=-\alpha\left(p_{1}-p_{2}\right) \kappa^{3 / 2}
$$

where

$$
\alpha=\sqrt{\frac{8 \pi \tilde{L}}{\tilde{N}_{A} \tilde{h}^{3}}} \lambda \tilde{a}^{3} \tilde{c}
$$

characterizes the rate of the fouling. The rate of change of permeability scales with the permeability to the power of $3 / 2$, as also shown in Bowen et al. for a standard-blocking configuration [20].

Equations (2) and (15) subject to conditions (3)-(8) and an initial condition for $\kappa$ forms a closed system for the velocities $u_{i}, v_{i}$ and pressures $p_{i}, i=1,2$, and the permeability $\kappa$.

\subsection{Model reduction}

Using (2b) and conditions (4) and (8) gives

$$
\begin{aligned}
& u_{1}=\frac{\partial p_{1}}{\partial x}\left(\frac{y^{2}}{2}-\frac{1}{2}\right), \\
& u_{2}=\frac{\partial p_{2}}{\partial x}\left(\frac{y^{2}}{2}-(1+\ell) y+\ell+\frac{1}{2}\right) .
\end{aligned}
$$

Equation (2a) subject to (5) then gives

$v_{1}=-\frac{\partial^{2} p_{1}}{\partial x^{2}}\left(\frac{y^{3}}{6}-\frac{y}{2}\right)$

$v_{2}=\frac{\partial^{2} p_{2}}{\partial x^{2}}\left(-\frac{y^{3}}{6}+\frac{1+\ell}{2} y^{2}-\left(\frac{1}{2}+\ell\right) y+\frac{1}{6}+\frac{\ell}{2}-\frac{\ell^{3}}{3}\right)$.

Finally, conditions (7) may be used with (19) to give

$$
\begin{aligned}
\frac{1}{3} \frac{\partial^{2} p_{1}}{\partial x^{2}} & =\kappa(x, t)\left(p_{1}(x, t)-p_{2}(x, t)\right), \\
\frac{\ell^{3}}{3} \frac{\partial^{2} p_{2}}{\partial x^{2}} & =\kappa(x, t)\left(p_{2}(x, t)-p_{1}(x, t)\right),
\end{aligned}
$$

subject to

$$
\begin{aligned}
p_{1}(0, t) & =1, & \frac{\partial p_{1}}{\partial x}(1, t) & =0, \\
\frac{\partial p_{2}}{\partial x}(0, t) & =0, & p_{2}(1, t) & =0 .
\end{aligned}
$$

We have thus reduced the problem to solving the system (20) subject to the boundary conditions (21), with $\kappa$ determined by (15).

\section{Results}

Beginning with an initially uniformly permeable membrane $\kappa(x, 0)=1$ we can solve the system (20) subject to (21) to determine the pressures and initial outflow:

$$
\begin{gathered}
p_{1}=\left[\cosh (\gamma)+\ell^{3}+\ell^{6} \cosh (\gamma(1-x))+\ell^{3} \cosh (\gamma x)\right. \\
\left.+\ell^{3}(1-x) \gamma \sinh (\gamma)\right]\left[\left(1+\ell^{6}\right) \cosh (\gamma)\right. \\
\left.+\ell^{3}(2+\gamma \sinh (\gamma))\right]^{-1} \\
p_{2}=\left[\cosh (\gamma)+\ell^{3}-\ell^{3} \cosh (\gamma(1-x))-\cosh (\gamma x)\right. \\
\left.\left.-\ell^{3}(1-x) \gamma \sinh (\gamma)\right)\right]\left[\left(1+\ell^{6}\right) \cosh (\gamma)\right. \\
\left.+\ell^{3}(2+\gamma \sinh (\gamma))\right]^{-1} \\
\left.v\right|_{y=1}=\frac{\ell^{3}\left(\ell^{3} \cosh (\gamma(1-x))+\cosh (\gamma x)\right)}{3\left(\left(1+\ell^{6}\right) \cosh (\gamma)+\ell^{3}(2+\gamma \sinh (\gamma))\right)}
\end{gathered}
$$




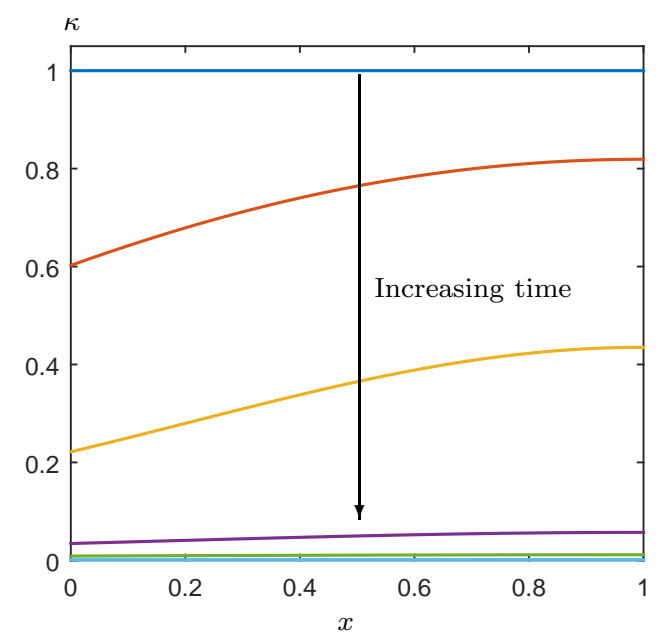

(a)

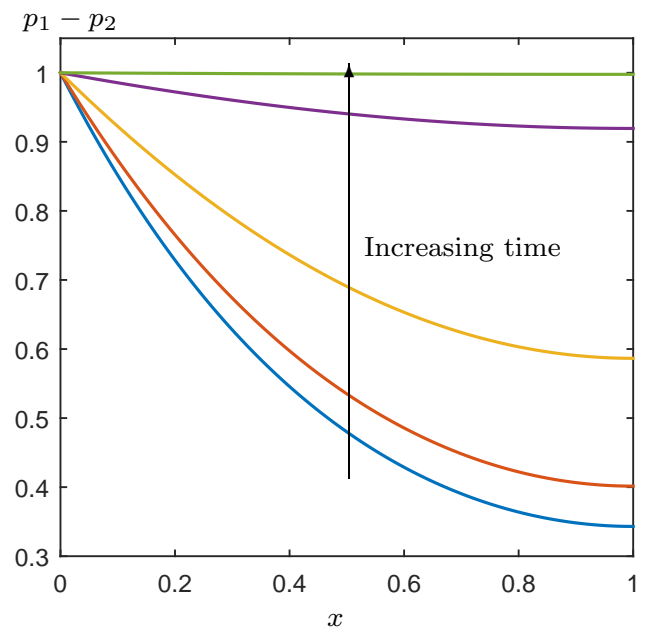

(b)

$v(x, 1, t)$

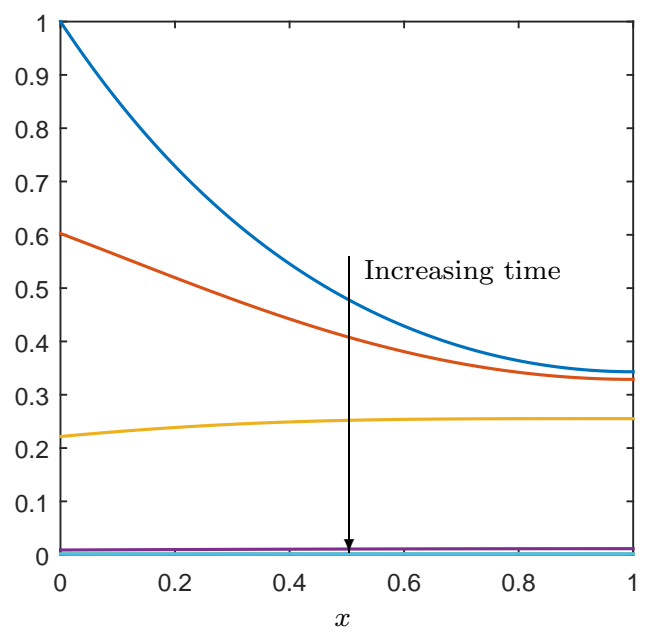

(c)

Figure 2: Evolution of (a) the wall permeability and (b) TMP $p_{1}-p_{2}$ and (c) permeate flux profile $\left(\left.v\right|_{y= \pm 1}\right)$, (2c), with time at $t=0,0.1,0.2,0.4,0.6,1$ for a single channel $(\ell=\infty)$ when $\kappa_{0}=1$ and $\alpha=1$. where $\gamma=\sqrt{3 \kappa_{0}\left(1+\ell^{3}\right) / \ell^{3}}$

As time progresses the pores will constrict as particles adhere to the membrane and the wall permeability will fall, obeying (15). In this case an analytic solution no longer exists and we must solve the system (20) numerically. The system was discretized using a finite-difference scheme in space and time and solved in MATLAB using the piecewise Chebychev polynomial interpolation Chebfun function (http://www.chebfun.org/, [21]).

When the channels appear in isolation (i.e., $\ell \rightarrow \infty$ ) the wall permeability initially falls close to the inlet, where the pressure difference is greatest and the flux across the membrane is higher (figure 2a). As the beginning of the membrane module blocks quickly, the fluid begins to favour transport through the unclogged membrane surface further down the channel, inducing a permeability decline that propagates down the channel. As time progresses the TMP rises, approaching a constant value (figure $2 \mathrm{~b}$ ).

The initial flux through the walls falls with distance from the inlet (figure 2c). Interestingly, as the membrane blocks the flux near the inlet drops more quickly, so the position of maximum outflow switches to further down the channel. This suggests that the outflow (which is the product of $\kappa$ and $p_{1}-p_{2}$, as given by (7)) is dominated by the TMP, $p_{1}-p_{2}$, to begin with and then influenced by the permeability, $\kappa(x, t)$, later. Such qualitative observations have also been reported during membrane fouling by cake filtration, where there is a critical time beyond which the cake resistance (which is manifested in the variation of permeability) dominates the filtration performance [22]. As $t \rightarrow \infty$ and the membrane blocks, the flux through the wall tends to zero everywhere.

When neighbouring channels are included there is a significant hydrodynamic interaction between the channels, which affects the pressure profile across the membrane. Now the flow is directed through the membrane walls not only close to the inlet but also near the capped end (figure 3c). As a result, the wall permeability falls most quickly in these areas, while the permeability in the centre remains higher (figure $3 \mathrm{a}$ ). The permeability profile exhibits a greater level of symmetry about the midpoint of the channel and so although the TMP still approaches unity across the channel length, it evolves in a more symmetric manner (figure 3b). As such, the filter blocks more uniformly across its length, leading to a more uniform wall flux with increasing time (figure 3c). We also note that the flux decline is faster in the early stages for the case of an isolated fibre but is faster in the later stages when neighbouring fibres are introduced.

\section{Module design optimisation}

For a given permeability $\kappa_{0}$ we are interested in the optimal fibre spacing that maximizes the instantaneous 


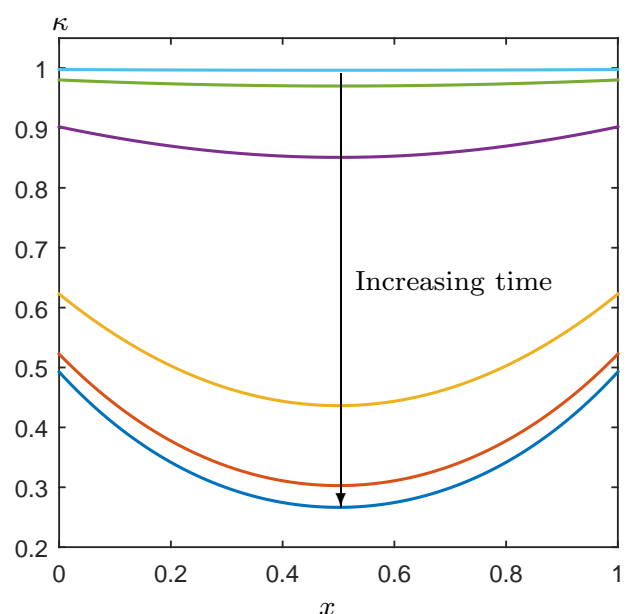

(a)

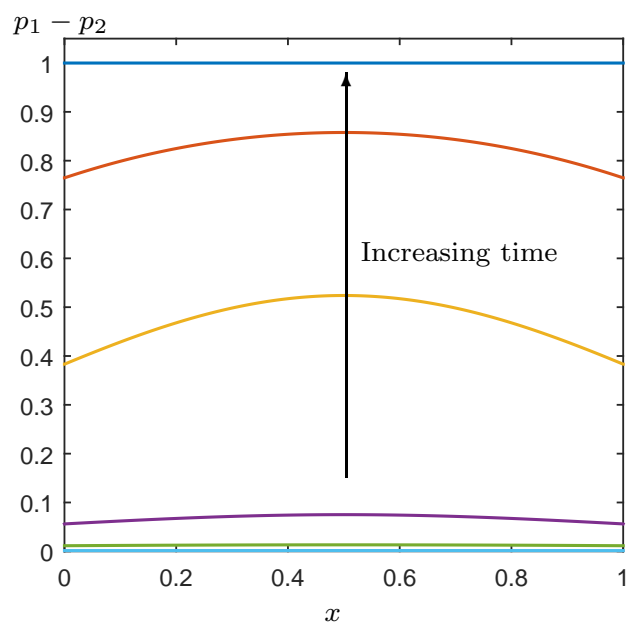

(b)

$v(x, 1, t)$

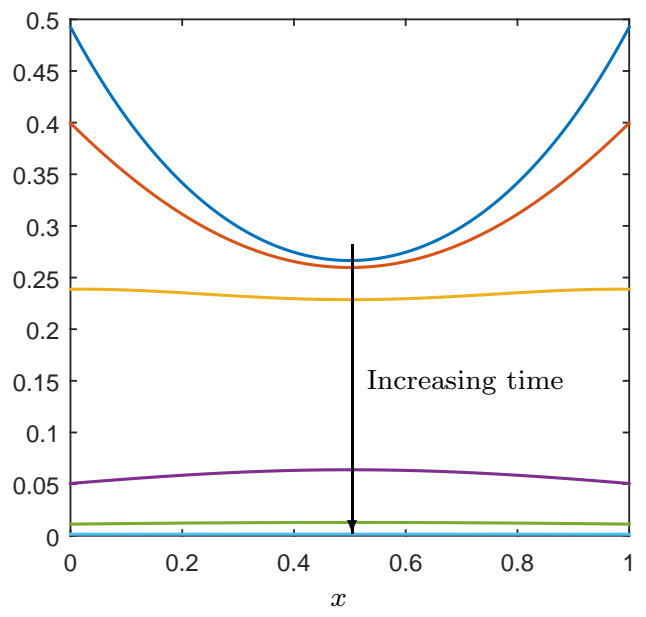

(c)

Figure 3: Evolution of (a) the wall permeability and (b) TMP $p_{1}-p_{2}$ and (c) permeate flux profile $\left(\left.v\right|_{y= \pm 1}\right)$, (2c), with time at $t=0,0.1,0.2,0.4,0.6,1$ for a device whose area occupied inside and outside the channels is equal $(\ell=1)$ when $\kappa_{0}=1$ and $\alpha=1$. flux per unit area occupied by the device, expressed as,

$$
\mathcal{Q}_{\ell}\left(t ; \kappa_{0}, \ell\right)=\frac{Q\left(t ; \kappa_{0}, \ell\right)}{1+\ell} .
$$

Here the flux through the system at time $t$ is given by

$$
Q\left(t ; \kappa_{0}, \ell\right)=2 \int_{0}^{1} u_{1}\left(0, y, t ; \kappa_{0}, \ell\right) \mathrm{d} y=-\frac{2}{3} \frac{\partial p_{1}\left(0, t ; \kappa_{0}, \ell\right)}{\partial x}
$$

using (17), and so

$$
\mathcal{Q}_{\ell}=-\frac{2}{3(1+\ell)} \frac{\partial p_{1}\left(0, t ; \kappa_{0}, \ell\right)}{\partial x} .
$$

Using (22) we can determine explicitly the optimal separation $\ell$ for a given $\kappa_{0}$ (figure 4 a inset). When $\kappa_{0}=1$ we find that the optimal fibre spacing that maximizes the initial flux per unit area, $Q_{\ell}(0 ; 1, \ell)$ is $\ell \approx 1.37$, indicating that approximately $30 \%$ more space should be occupied outside the channel than inside (figure $4 \mathrm{a}$ ). The change in $\mathcal{Q}_{\ell}(0 ; 1, \ell)$ with $\ell$ is greater when the separation distance is less than the optimum value compared with when $\ell$ exceeds the optimum value, indicating that it is better to overestimate the optimum packing fraction than underestimate.

However, the metric $\mathcal{Q}_{\ell}\left(0 ; \kappa_{0}, \ell\right)$ only takes into account the initial filtration behaviour. When we incorporate the subsequent effects of fouling then it is the overall fluid processed that takes precedent over the instantaneous flux. Since there are a wide variety of applications for direct-flow filtration there is no single metric that is able to capture filtration optimization when membrane blocking is important. However, two typical features that are commonly desirable to maximize are either (i) the total volume of fluid that has been processed by the device after a set time; or (ii) the total volume of fluid that has been processed before the flux falls to a given fraction of its initial value (at which point the process ceases and the filter is changed). The former is more desirable when the filtered material needs to be processed as fast as possible and membrane costs are minimal; the latter is typically required when the membrane device that is used is expensive since this allows the most fluid to be processed before the filter reaches the end of its useful lifetime.

To characterize property (i) we consider a metric $\mathcal{V}_{T}$, defined to be the total volume of fluid processed per unit area occupied by the system, after a fixed time, $T$ :

$$
\mathcal{V}_{T}=\frac{1}{(1+\ell)} \int_{0}^{T} Q \mathrm{~d} t^{\prime}=-\frac{2}{3(1+\ell)} \int_{0}^{T} \frac{\partial p_{1}}{\partial x} \mathrm{~d} t^{\prime} .
$$

using (17).

Property (ii) is captured through the metric $\mathcal{V}_{\beta}$, defined to be the total volume of fluid processed per unit volume occupied by the system when the flux has fallen to a fraction, $\beta$, of its original value:

$$
\mathcal{V}_{\beta}=\frac{1}{1+\ell} \int_{0}^{t^{*}} Q \mathrm{~d} t^{\prime}-\frac{2}{3(1+\ell)} \int_{0}^{t^{*}} \frac{\partial p_{1}}{\partial x} \mathrm{~d} t^{\prime},
$$


where $t^{*}$ is given implicitly by

$$
\frac{Q\left(t^{*} ; \kappa_{0}, \ell\right)}{Q\left(0 ; \kappa_{0}, \ell\right)}=\beta,
$$

which may be written as

$$
\frac{\partial p_{1}\left(0, t^{*} ; \kappa_{0}, \ell\right)}{\partial x}=\beta \frac{\partial p_{1}\left(0, t^{*} ; \kappa_{0}, \ell\right)}{\partial x},
$$

using (17).

When maximizing for the total fluid processed after a given time $\left(\mathcal{V}_{T}\right)$, we find that the faster the membrane blocks due to the contaminants, the closer the fibres should be packed (figure $4 \mathrm{~b}$ ). We have seen that packing fibres more closely together lowers the velocity across the membrane (figure 2c, 3c). When the membrane blocks, the initial uneven distribution in the velocity across the walls is smoothed out with time since blocking occurs more quickly in those regions where the permeate flow rate is faster. This has the result of improving the uniformity in the flow across the membrane, and thus counteracting the main disadvantage of packing fibres closer together. This indicates that we should pack fibres more closely into direct-flow devices when the contaminant concentration in the feed solution is higher.

If the desired outcome is to maximize the volume of processed fluid before the filter reaches the end of its useful lifespan, $\mathcal{V}_{\beta}$, contrary to the case where we wish to maximize $\mathcal{V}_{T}$, the optimal separation now increases with increasing fouling rate $\alpha$ (though the effect is minimal and the optimal spacing does not deviate too far from unity) (figure 4c). This indicates that the device should be composed of approximately equal internal and external space when $\kappa_{0}=1$.

\subsection{Variation of optimum separation with initial perme- ability}

The module design specification is dependent on the initial permeability of the membrane, since this affects the hydrodynamic pressure profiles in the device. As the initial permeability increases the membrane becomes more porous, or open. As a result, the local TMP $\left(p_{1}-p_{2}\right)$ decreases, which indicates that separating the fibres will reduce $\tilde{p}_{2}$, enhance the local TMP, and thus increase the wall flux $\left.v\right|_{y=1}$. Thus we observe that the optimum fibre separation distance increases with permeability (figure 5a). As discovered in figure $4(\mathrm{~b}, \mathrm{c})$, the spacing $\ell_{T}^{*}$ that optimizes $\mathcal{V}_{T}$ reduces with increasing fouling rate $\alpha$, while the spacing $\ell_{T}^{*}$ that optimizes $\mathcal{V}_{\beta}$, increases with the rate of fouling, though only marginally.

\subsection{Dynamic design optimisation}

An optimum fibre separation exists that maximizes the total volume processed, but it is sensible to ask the question whether we can improve on the filtration efficiency even further by changing the fibre separation distance with time. To maximize the instantaneous flux per unit area

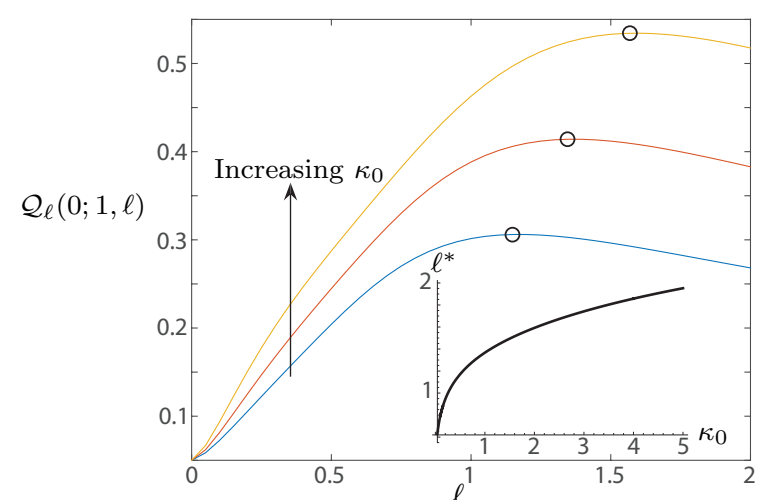

(a)

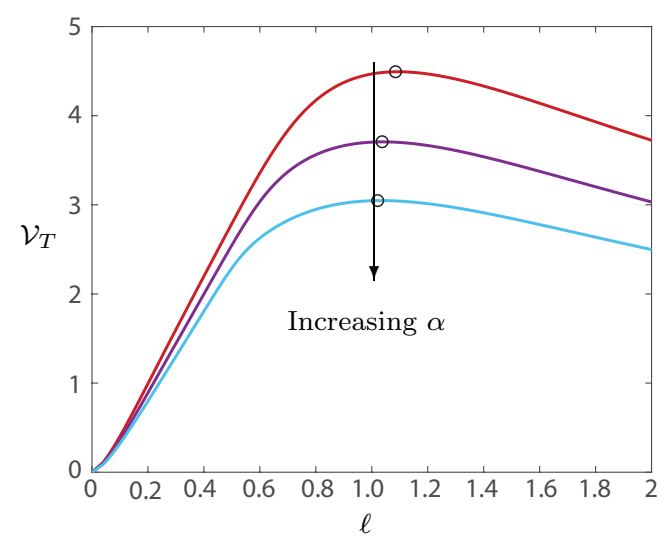

(b)

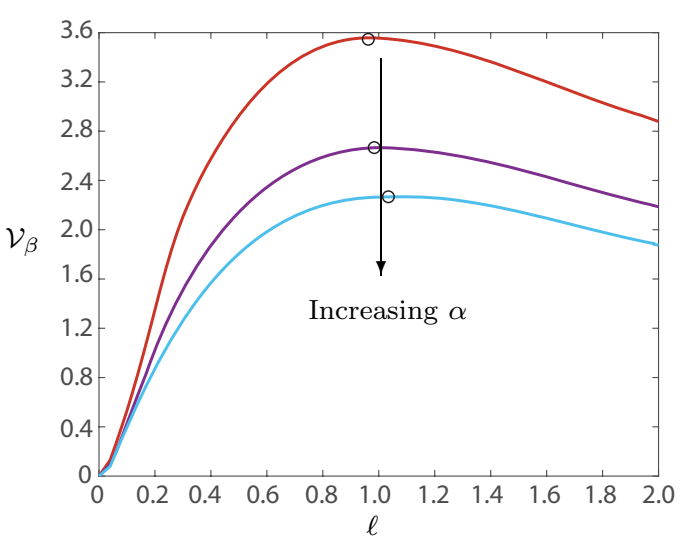

(c)

Figure 4: (a) Variation of $\mathcal{Q}_{\ell}$, the flux processed per unit time per unit area occupied by the direct-flow device, with inter-channel spacing, $\ell$, in the absence of fouling $\alpha=0$ for $\kappa_{0}=0.5,1,2$. Inset: Variation of the optimal spacing $\ell^{*}$ that maximizes $\mathcal{Q}_{\ell}$ (b) Variation of $\mathcal{V}_{T}$, the total volume of fluid processed per unit area occupied by the system after a fixed time $T=1.5$, with $\ell$ when $\alpha=0.5,1,1.5$ and $\kappa_{0}=1$ (c) Variation in $\mathcal{V}_{\beta}$, the total volume of fluid processed per unit area occupied by the system when the flux has fallen to a fraction $\beta=0.1$ of its original value, with $\ell$, when $\alpha=0.5,1,1.5$ and $\kappa_{0}=1$. The circles highlight the 


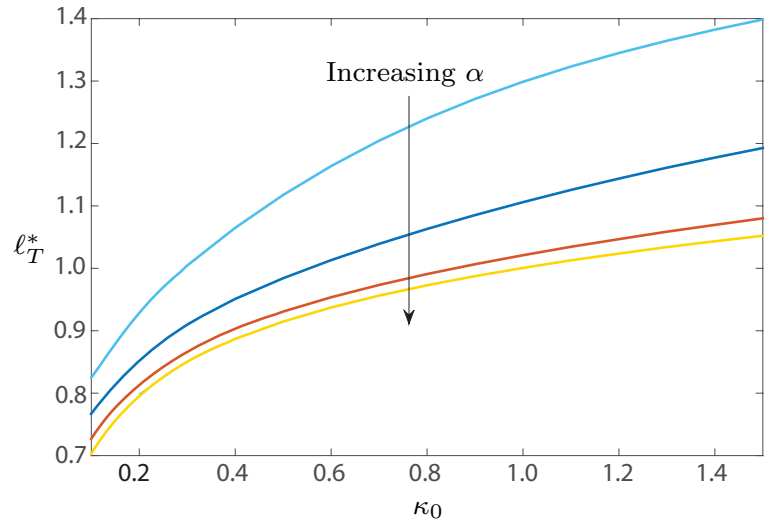

(a)

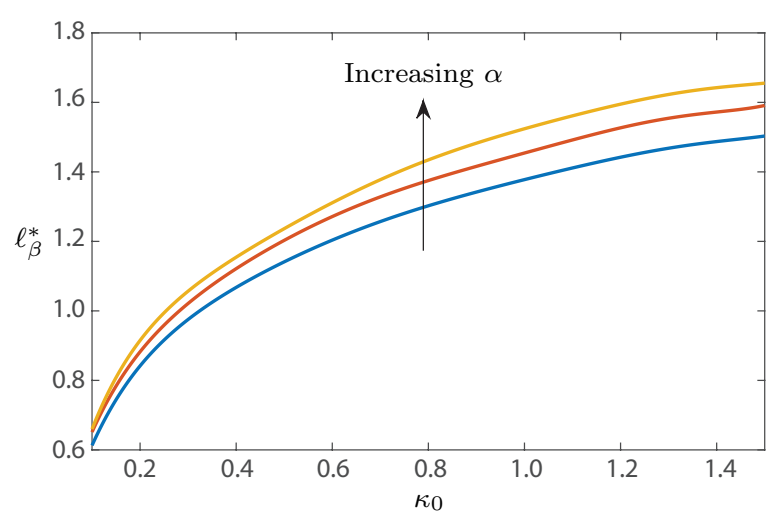

(b)

Figure 5: (a) Dependence of the separation distance, that maximizes $\mathcal{V}_{T}\left(\ell_{T}^{*}\right)$ on the initial membrane permeability $\kappa_{0}$ when $T=1.5$ and $\alpha=0.1,0.5,1,1.5$. (b) Dependence of the separation distance that maximizes $\mathcal{V}_{\beta}\left(\ell_{\beta}^{*}\right)$ on the initial membrane permeability $\kappa_{0}$ when $\beta=0.1$ and $\alpha=$ $0.5,1,1.5$. occupied at each time, $\mathcal{Q}_{\ell}\left(t ; \kappa_{0}, \ell\right)$, we find that we must reduce the separation distance $\ell$ as time progresses (figure 6). Physically this could be achieved by squeezing together the fibres by bundling them inside a flexible or soft cartridge. However, this may be quite complicated to achieve both from a fabrication and operational perspective. As a result, we must weigh the advantages against the improvement in performance.

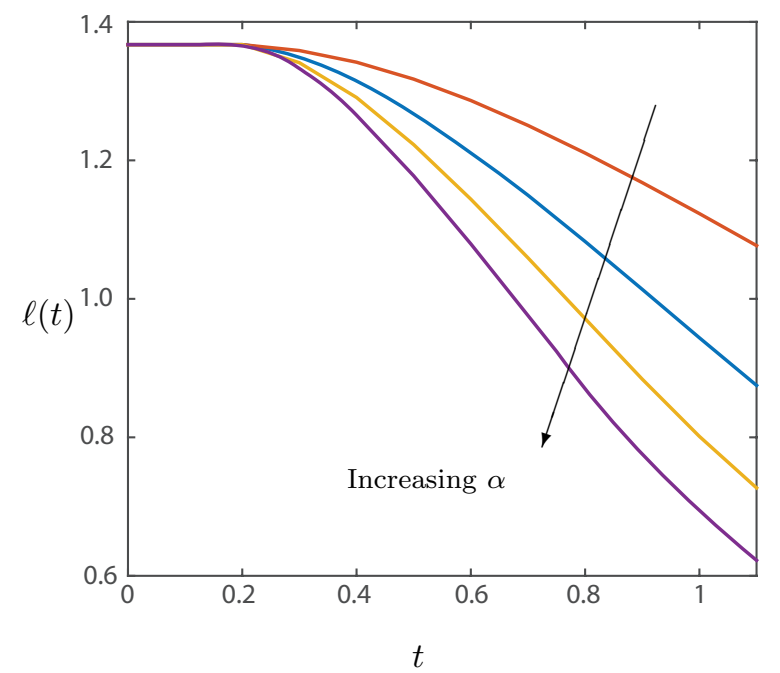

Figure 6: The time-dependent spacing parameter, $\ell(t)$, that optimizes the instantaneous flux per unit area $\mathcal{Q}_{\ell}\left(t ; \kappa_{0}, \ell(t)\right)$, when the initial permeability $\kappa_{0}=1$ and fouling rate $\alpha=0.5,1,1.5,2$.

Choosing the inter-fibre spacing that maximizes the total volume processed over that entire given time (i.e., $\ell_{T}$ ) offers a significant improvement when compared with the constant choice for $\ell$ that maximizes the initial flux per unit area (for instance, giving an approximately $6 \%$ improvement when $\kappa_{0}=1, \alpha=1$ and $\left.T=1.5\right)$. Dynamically changing the inter-fibre spacing $\ell(t)$ offers a small additional improvement over this case (approximately $1.4 \%$ when $\alpha=0.5,2 \%$ when $\alpha=1$ and $2.7 \%$ when $\alpha=2$ ). Although the additional benefits are unlikely to justify the extra manufacture and operating complications of a dynamically adjusting inter-fibre spacing, in instances where the membrane is very expensive to manufacture this may still be a worthwhile improvement. 


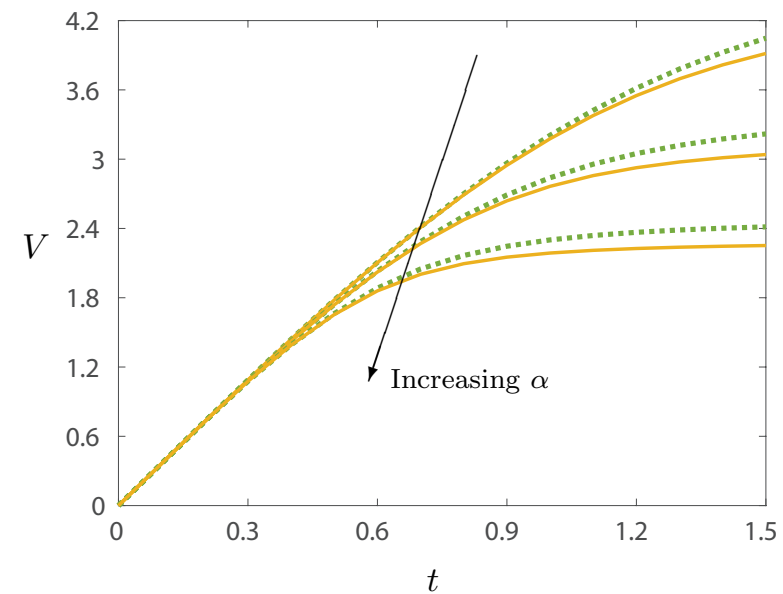

Figure 7: Total volume processed, $V=\int_{0}^{t} Q \mathrm{~d} t^{\prime}$ versus time for the constant fibre spacing that maximizes $V_{T}$ $\left(\ell_{o p t_{T}}\right)$ for $T=1.5$ (solid yellow curve) and the dynamic spacing $\ell(t)$ that optimizes the instantaneous flux (dashed green curve) when $\kappa_{0}=1$ and $\alpha=0.5,1$ and 2 .

\section{Conclusions}

We have shown and quantified the role that membrane clogging plays in direct-flow filtration and the design optimization that arises as a result. We considered an idealized direct-flow filter composed of a series of permeable fibres packed into a single device with equal inter-fibre spacing and showed that an optimal separation of the individual fibres was shown to exist. Choosing to optimize the total fluid filtered over a filtration run yielded improvements of around to $6 \%$ compared with the fibre spacing chosen to maximize the flux initially. Dynamically controlling the fibre separation was found to give an additional $2 \%$ improvement. Although this perhaps does not justify the extra manufacture and operating effort, in instances where maximizing the filtered fluid is the main priority it may be worth the extra effort.

In all of the modelling presented here we assume that the fibres are uniformly spaced. In practice there will invariably be some randomness in the inter-fibre spacing. However, we expect that such irregularities will smooth out on average so that our analysis provides the typical expected behaviour of a device. The fibres were also assumed to be rigid. Recent studies illustrate that the fibres may deform when high transmembrane pressures are applied, leading to increased fouling as the pore sizes increase [23]. An interesting generalization would thus be to study the effect that this has on the resulting behaviour, and in particular how this adjusts the optimum packing fraction.

The work that we have presented here offers a simple framework that is readily adapted to describe a specific modelling challenge, either by appropriate choices in the system parameters in this model or by simple model generalizations. An interesting extension of the work would be to analyse the influence of multiple-sized solutes on the agglomeration and fouling of the membrane module and subsequent impact on the throughput, particularly when the disparity in particle sizes is such that some particles are small enough to penetrate the membrane while others collect as a 'cake' layer on the membrane surface. The present analysis offers the potential for design improvement in both existing and new direct-flow filtration devices with open porous membranes.

\section{Acknowledgements}

MW acknowledges the support from ENSTA ParisTech for providing the opportunity of a research internship at the Mathematical Institute, University of Oxford. SM is grateful to EPSRC and the Royal Society. IMG gratefully acknowledges support from the Royal Society through a University Research Fellowship.

\section{References}

[1] Winston Ho and Kamalesh Sirkar. Membrane handbook. Springer Science \& Business Media, 2012.

[2] K. Sutherland. Profile of the International Membrane Industry - Market Prospects to 2008. Elsevier Science, 2003.

[3] G. Belfort. Synthetic Membrane Process: Fundamentals and Water Applications. Water pollution. Elsevier Science, 2012.

[4] R. D Noble and S. A. Stern. Membrane Separations Technology: Principles and Applications, volume 2. Elsevier, 1995.

[5] S. Karode. Laminar flow in channels with porous walls, revisited. J. Membr. Sci, 191(1-2):237-241, 2001.

[6] J. G. Herterich, Q. Qu, R. W. Field, D. Vella, and I. M. Griffiths. Optimizing the operation of a direct-flow filtration device. J. Eng. Math. (to appear), 2016.

[7] G. K. Pearce. UF/MF Membrane Water Treatment: Principles and Design. Water Treatment Academy Bangkok, 2011.

[8] M. F. Hurwitz. Flows Through Porous Tubes-The End Cap Problem. Proc. Mathematical Problems in Industry, 1989.

[9] S. Chang, A. G. Fane, and T. D. Waite. Analysis of constant permeate flow filtration using dead-end hollow fiber membranes. J. Membr. Sci., 268(2):132-141, 2006.

[10] C. Serra, M. J. Clifton, P. Moulin, J.-C. Rouch, and P. Aptel. Dead-end ultrafiltration in hollow fiber modules: Module design and process simulation. J. Membr. Sci., 145(2):159-172, 1998.

[11] P. Sanaei, G. W. Richardson, T. P. Witelski, and L. J. Cummings. Flow and fouling in a pleated membrane filter. J. Fluid Mech., 795:36-59, 2016.

[12] B. Van der Bruggen, C. Vandecasteele, T. Van Gestel, W. Doyen, and R. Leysen. A review of pressure-driven membrane processes in wastewater treatment and drinking water production. Environ. Progress, 22(1):46-56, 2003.

[13] J. G. Herterich, I. M. Griffiths, R. W. Field, , and D. Vella. The effect of a concentration-dependent viscosity on particle transport in a channel flow with porous walls. AIChE J., 60(5):18911904, 2014.

[14] O. Kedem and A. Katchalsky. Thermodynamic analysis of the permeability of biological membranes to non-electrolytes. Biochim. et Biophys. Acta, 27:229-246, 1958.

[15] G. S. Beavers and D. D. Joseph. Boundary conditions at a naturally permeable wall. J. Fluid Mech., 30:197-207, 1967.

[16] R J. Shipley, S. L. Waters, and M. J. Ellis. Boundary conditions at a naturally permeable wall. Biotechnol. Bioeng., 107:382$392,2010$.

[17] Altena F. W. and G. Belfort. Lateral migration of spherical particles in porous flow channels: Application to membrane filtration. Chem. Eng. Sci., 39(2):343-355, 1984. 
[18] J. R. Ockendon and Ockendon H. Viscous Flow, volume 13. Cambridge University Press, 1995.

19] R. W. Field, D. Wu, J. A. Howell, and B. B. Gupta. Critical flux concept for microfiltration fouling. J. Membr. Sci., 100(3):259 272, 1995.

[20] W. R. Bowen, J. I. Calvo, and A. Hernandez. Steps of membrane blocking in flux decline during protein microfiltration. J. Membr. Sci., 101(1):153-165, 1995.

[21] T. A. Driscoll, N. Hale, and L. N. Trefethen. Chebfun and Approximation Theory. Pafnuty Publications, Oxford, 2014.

[22] S. Mondal and S. De. Generalized criteria for identification of fouling mechanism under steady state membrane filtration. J. Membr. Sci., 344(1):6-13, 2009.

[23] Q. She, X. Jin, and C. Y. Tang. Osmotic power production from salinity gradient resource by pressure retarded osmosis: effects of operating conditions and reverse solute diffusion. J. Membr. Sci., 401:262-273, 2012. 